\title{
Déambulation et quête de soi dans Les Tribulations du dernier Sijilmassi de Fouad
} Laroui

\section{Strolling and Self-quest in The Tribulations of the Last Sijilmassi by Fouad Laroui}

\author{
Hafid ABOUELKACEM ${ }^{1}$ (D)
}

'PhD Candidate, Ibn Zohr University, Faculty of Letters and Human Sciences, Department of French Language and Literature, Agadir-Morocco

\section{ORCID: H.A. 0000-0003-4391-8658}

\section{Corresponding author:} Hafid ABOUELKACEM,

Université lbno Zohr, Faculté des Lettres et des Sciences Humaines, Département de langue et littérature françaises,

Agadir-Maroc

E-mail: haafidabouelkacem@gmail.com

Submitted: 28.01 .2021

Revision Requested: 12.04.2021

Last Revision Received: 18.04.2021

Accepted: 04.05.2021

Citation: Abouelkacem, H. (2021). Déambulation et quête de soi dans les Tribulations du dernier Sjilmassi de Fouad Laroui. Litera, 31(2), 633-652. https://doi.org/10.26650/LITERA2021-870270

\section{RÉSUMÉ}

Cette étude a pour ambition de mettre en lumière les tribulations du marcheur dans ce roman philosophique de Fouad Laaroui. En cela que nous avons explicité comment Adam connait une métamorphose en se vidant l'esprit de tous les textes et fragments poétiques de littérature française qu'il avait auparavant mémorisé. En montrant que dans la marche, le corps et l'esprit sont en plein dynamisme. La métamorphose ne s'est pas faite sans malaise, sans vertige identitaire du fait que le "flot de mots " s'accompagne de "maux ». Notre marcheur se perd dans ses pensées une fois dans la marche. II y perd ses repères et se met en quête de soi. À cela s'ajoute, une sensation du dédoublement et de malaise très manifeste chez le marcheur. La pratique de la marche apparaît aussi comme une révolte et résistance face à la vitesse de la vie moderne. La décision de marcher apparaît comme une folie du fait que choquante et perçue comme acte transgressif. Marcher pour Adam c'est exister et résister. le protagoniste entend se « tenir hors » de la vitesse en ce qu' il éprouve un désir de passer d'un lieu profane (la ville) à un lieu sacré.

Mots-clés: Marche, tribulation, quête de soi, corps, déambulation

\section{ABSTRACT}

This study aims to highlight the tribulations of the walker in the philosophical novel The Tribulations of the Last Sijilmassi by Fouad Laaroui. We explain how Adam experiences a metamorphosis by emptying his mind of all the texts and poetic fragments of French literature that he had previously memorized. When walking, the body and mind are in full dynamism. The metamorphosis is not without unease and identity vertigo, because the "flow of words" is accompanied by headache. Our walker gets lost in his thoughts once he is on a walk. In walking, there is a loss of landmarks and of self-seeking. In addition, a feeling of duplication and discomfort is evident in the walker. The practice of walking also appears as a revolt against and resistance to the speed of modern life. The decision to walk appears to be a folly because it is shocking and perceived as a transgressive act. For Adam, to walk is to exist and resist. The protagonist intends to "hold himself out" of the speed of modern life, in that he feels a desire to move from a profane place (the city) to a sacred place. Keywords: Walking, tribulation, self-quest, body, strolling 


\section{EXTENDED ABSTRACT}

Walking is understood by many thinkers as "a call to self-transformation." For Adam, walking is first and foremost a way of knowing oneself. The ordeal of the road offers him the opportunity for an experience that he deems necessary to identify himself. His walk appears to be both a quest and an escape. Indeed, he is caught in a simultaneous movement of distance and approach to a place. He flees the city and the speed that is the basis of his daily life, to embark on a long march towards Azemmour, the place of his ancestors. If Adam flees from speed, it is not only because it causes him "headaches;" it is also because he wants, through this act, to "deviate from the right path, the path of the predictable, of the given in advance as sure." Adam deviates from the right path when he abandons everything, including his wife and his work. Walking not only stimulates his body, it also acts on his mind to the point where he indulges in multiple philosophical reflections. Urban life and the speed on which it is founded become for Adam threatening and disturbing. They stir up the flow of words, which overwhelms him as soon as he starts walking. Our subject empties his mind of extraordinary logorrhea of French literature, which causes a "headache" and thus complicates his life.

In The Tribulations of the Last Sijilmassi, Adam, as a walker, travels through the city his psychic absences, lost in the labyrinth that his thoughts form. There is in Adam a feeling of uncertainty and ambiguity that we want to see resolved. It is easy to see that his mind is indeed dynamic, because it is always in a state of absence, absent because it is thinking until it enters a "trance." In particular, he exerts himself to hurtle down the stairs after a consultation with a psychiatrist on the nature of his absences and the radical change he has opted for.

Strictly speaking, the practice of walking is also part of the urban space. In this space, the walker struggles, with the intention of transgressing established norms. Thus, his ambition is to go beyond "civilized spaces" for a wider understanding of the world. In other words, walking means revolt against the norms established by society in that this act constitutes a favored path to reweave lost ties. It is clear to us that the march constitutes a moving critique of modernity. After an epiphany experienced over the Andaman Sea, he revolts against the speed that is the foundation of the contemporary world. As a result, he begins to avoid all machinery, as speed causes him vertigo. His decision to reach the city by walking seems to be out of the ordinary, which leads to 
his returning home in the custody of two gendarmes. This is how his walk begins, a walk that later awakens his reflection on the world.

The spiritual dimension of the walk highlights the passage of the walker from a profane place to a sacred place. It is clear that the walker is trying to increase the arduousness of the journey towards his or her birthplace, which is perceived as sacred and as a place of healing. For our subject, it is essential to walk until his arrival, otherwise it is death that awaits him. In this respect, if, in walking, the body can be in full dynamism, the spirit is also subjected to a severe test.

In addition to this, poetic fragments that the memory of the walker had stored up emerge in waves. It's as if he empties himself of poetic fragments to change into an authentic Moroccan whom he should never have abandoned. This is how walking becomes synonymous with invention. In coming face-to-face with the world, we can say that our walker intends to "stand out." In this way, walking also becomes synonymous with existence, which means resisting modern life and the speed on which it is based. If not, life gets bogged down. 


\section{Introduction}

Lauréat du prix Jean Giono en 2014, Les Tribulations du dernier Sijilmassi (2014) est I'histoire d'un ingénieur « Adam Sijilmassi » qui vécut des tribulations de l'esprit et des aventures, au sens d'épreuves traversées par l'esprit notamment dans la façon de voir le monde. C'est le roman de l'épiphanie et d'un choc vécu au-dessus de la mer d 'Andaman. C'est le récit du questionnement absurde et de l'esprit errant où la raison de cet homme agnostique se brise par la vitesse hallucinante du Boeing, le poussant à se poser la question « qu'est-ce que je fais ici ? » (Laroui, 2014. p.6). L'écrivain maroconéerlandais met en lumière un contraste entre la vie que mène Adam Sijilmassi et la vie que menaient ses ancêtres. C'est aussi pour dire quelle révélation a reçu le protagoniste et quel homme il est devenu après cette épiphanie vécue. Outre sa décision de marcher et de se mettre en dehors de la vitesse et comment il passe pour un fou, l'auteur met en lumière révolte et résistance ainsi que nostalgie : révolte et résistance par la marche contre la vitesse qui fonde la vie moderne, nostalgie par la volonté de vivre à la manière de ses ancêtres d'Azemmour. Au fil du texte de Laroui se dessine un itinéraire, un parcours en marge, celui d'Adam qui s'aventure par la marche et part en quête de ses origines. Partant du fait que dans la marche, l'esprit, le corps et le monde se répondent, et que le corps et l'esprit sont du même degré d'activité, nous tenterons de mettre en lumière les tribulations de l'esprit et les aventures du héros dans le roman. Au commencement, un survol théorique des études faites sur la marche est indispensable. En cela que nous allons recourir notamment aux études anthropologiques axées sur la marche en l'occurrence, ceux de Rebecca Solnit et David Le Breton. Il s'agira ensuite de présenter l'apport du philosophe Michel de Certeau sur le rapport entre la marche et la pensée. Il est de circonstance de se demander comment la marche d'Adam devient un appel à la métamorphose de soi et une tentative de changer d'existence. Nous allons mettre en lumière la marche et le dynamisme auquel elle donne naissance à travers le cheminement d'Adam. Ensuite, nous mettrons en évidence comment la marche devient dans ce roman à la fois révolte, résistance et nostalgie. Enfin, on verra également comment le sujet marcheur passe d'un lieu profane à un lieu qui lui est sacré.

\section{Considérations théoriques}

Il est bien difficile de cerner la marche, lui imposer une signification unique du fait de sa nature mouvante. Elle apparait comme une pratique banale, insaisissable et complexe. Toutefois, il est indispensable de faire appel à de multiples disciplines à titre 
d'exemple : I'anthropologie, la philosophie si l'on veut approcher la marche dans la littérature. L'une des premières figures ayant approché cette pratique est l'écrivaine américaine Rebecca Solnit qui adopte dans son livre L'art de marcher une approche sociologique et anthropologique. Elle voit que « la marche a généré le sentiment de l'espace » (Solnit, 2002, p. 10). Et l'anthropologue de poursuivre qu'« idéalement, marcher est un état où l'esprit, le corps et le monde se répondent » (Solnit, 2002, p. 12). Pour elle, marcher et penser sont difficilement dissociables, c'est ainsi que le corps et l'esprit seraient d'un même degré d'activité, et ce, harmonieusement, afin que la déambulation ait lieu. C'est notamment aux travers de la marche que la voie s'ouvre aux sens et à la sensation. L'anthropologue David Le Breton dit dans ce sens que la marche fait «plonge[r] [le marcheur] dans une forme active de méditation sollicitant une pleine sensorialité » (Le Breton, 2000, p. 11). Et l'anthropologue d'ajouter que la marche se veut une perte des repères et une quête de soi, une façon de se rassembler soi (Le Breton, 2000, p. 11) qui va à l'encontre de toute activité devant remplir une fonction. Il conçoit donc la marche comme une activité impliquant à la fois le rôle d'acteur et de spectateur. Dans ce sens, le sujet marcheur agit de corps et d'esprit, assiste au spectacle du paysage pour ainsi le créer à son tour. C'est ainsi que dans sa marche, il éprouve le désir de comprendre le monde qui l'entoure et pas seulement pour chercher un point d'arrivé. II poursuit sa réflexion en avançant que la posture du marcheur doit être celle de la disponibilité totale au monde (Le Breton, 2000, p. 22). Ainsi, le marcheur doit être en disponibilité totale face à la nature. Pour ce faire, un certain équilibre lui est indispensable entre le corps et l'esprit. Cela montre que dans la marche, le mouvement du corps stimule celui de l'esprit. Pour le sujet marcheur, l'expérience de l'espace terrestre passe inéluctablement par le corps avant d'être intellectualisée, conceptualisée. Rebecca Solnit pour sa part explicite l'importance de la perception qui se réalise par l'entremise du corps et de la marche comme une création en soi. «La marche elle-même étend le corps au monde, à l'instar des outils qui le prolongent. Le chemin est un prolongement du marcheur [...] On trouve donc trace du corps qui marche dans les lieux qu'il a créés » (Solnit, 2002, p. 47).

Il importe de souligner que le corps en marche peut être saisi comme entremise entre l'esprit et le monde. Cet échange constant qui se fait par l'entremise du corps entre l'esprit et le monde nous est d'importance en ce qu'il permet de mettre en lumière la marche dans le texte littéraire. En effet, l'écriture et la marche ont de profondes similitudes en ce qu'elles constituent « un appel à la métamorphose de soi » (Le Breton, 2000, p. 164) autrement, à se recréer un soi détaché des autres et ayant la capacité de renaitre sous des formes variées. 
La déambulation fait que le sujet marcheur se promène non seulement dans l'espace c'est aussi dans sa mémoire, en méditant sur ses souvenirs. Toutefois, il est au cœur d'une mémoire collective. L'identité qui se rapporte à l'espace montre au sujet marcheur qu'il est relié à d'autres à travers les signes qu'il capte. En cela que le marcheur se livre à un travail de déchiffrage des signes que l'espace émet le long de son cheminement. C'est donc ces signes-là qui seront symbolisés dans l'écriture pour refaire le voyage, se perdre dans des labyrinthes dans l'intention de recréer le monde. L'anthropologue considère que l'écriture peut être saisie comme mémoire des évènements innombrables cueillis au fil du chemin (Le Breton, 2000, p. 94).

Quant au philosophe Michel de Certeau, il avance l'idée que le corps et l'esprit sont indissociables. II dit dans ce sens : " penser, c'est passer; c'est interroger un ordre, s'étonner qu'il soit là, se demander ce qui l'a rendu possible, chercher en parcourant ses paysages les traces des mouvements qui l'ont formé, et découvrir dans ces histoires supposées grisantes comment penser autrement »(De Certeau, 1987, p. 51). André Carpentier propose d'inverser ces deux verbes dans l'acte déambulatoire, pour lui, passer c'est penser, du simple fait que «le corps et l'esprit agissent en complices dans le rapport au lieu » (Carpentier, 1987, p. 48). Et le philosophe d'expliciter : les «énonciations piétonnières » (De Certeau cité par Carpentier 2001, p. 148) qu'il conçoit comme réseaux de significations existant explicitement entre l'acte de marcher, l'acte de parler et celui d'écrire. Cela va de soi avec la déambulation littéraire qui peut être saisie comme mouvement indissociable de la marche, de la pensée et de l'écriture. Il y a donc là une dynamique plurielle qui fonde la marche dans la littérature.

Il peut y avoir des marcheurs contemporains qui se placent en position de révolte contre la culture, et les normes urbaines établies par l'homme moderne. C'est dans cet esprit qu'Alexis I'Allier avance l'idée que le marcheur solitaire marche souvent à contrecourant de tout un chacun, recherche une place dans la nature (L'Allier, 2004, p. 25). C'est ainsi que l'on peut affirmer que la marche constitue une critique en mouvement de la modernité. Dans le même ordre d'idée, Sylvain Tesson affirme dans ce sens que marcher, c'est fuir le monde numérisé et s'opposer ainsi au règne de la prévisibilité. Pour le critique littéraire Alexis l'Allier, dans la littérature, la déambulation s'inscrit d'abord dans l'espace urbain, cela peut être expliqué autant par une volonté de résistance face à la déshumanisation du monde que par le désir de comprendre ce monde déconstruit, du fait que le sujet marcheur, stipule l'Allier, est constamment en quête de sens (L'Allier, 2004, p. 33). L'anthropologue Le Breton voit dans ce sens que la marche 
"dans le monde contemporain, pourrait évoquer une forme de nostalgie ou résistance » (Le Breton, 2000, p. 14). Cette pratique qui apparait comme risible et désuète à cote des moyens de transport (avions, trains, métros..) est bien importante en ce qu'elle est valorisée notamment par les mouvements sociaux et culturels en tant que manifestation d'un droit à l'espace, loisir, retour à la nature ou comme quête spirituelle. On peut dire que La mise en marche dans la ville signifie tenter de «changer d'existence » (Le Breton, 2000, p. 25) de sortir de soi ainsi que d'entrer dans le labyrinthe, dans l'intention de mieux connaitre soi ou rentrer chez soi (Le Breton, 2000, p. 34).

L'acte de marcher contient en lui une dimension spirituelle qui se manifeste explicitement dans le pèlerinage, parce que caractérisé par le passage d'un lieu profane à un lieu sacré. Cela signifie que l'espace se scinde en deux. C'est ainsi que l'on peut avancer l'idée que le marcheur pèlerin prend son départ d'un lieu-dit profane qui peut être la ville, lieu représentant le confort et la sécurité, très souvent douloureusement et dans une extrême lenteur comme ayant des cailloux dans les souliers. L'anthropologue Le Breton met en lumière l'idée que les marcheurs pèlerins s'efforcent d'augmenter la pénibilité du voyage (Le Breton, 2000, p. 69) en direction d'un lieu qu'ils qualifient de sacré. L'anthropologue souligne également l'idée que le sujet marcheur « n'élit pas de domicile dans l'espace, mais bien dans le temps » (Le Breton, 2000, p. 26) On peut affirmer que ce n'est pas les frontières spatiales qui scinde le parcours du marcheur mais bien le cycle du jour et de la nuit. En cela qu'il se libère de tout obstacle spatial pour affronter les contraintes temporelles de la nature.

Le Breton stipule également que la pratique de la marche se présente comme une recherche de contemplation, d'abandon, de flânerie que la présence d'un compagnon briserait, contraignant à la parole, au devoir de communiquer (Le Breton, 2000, p. 3738). Il est d'évidence que le sujet marcheur fuit les hommes qui, assurément accablent les philosophes. C'est le cas du protagoniste du roman qui fuit les hommes parce qu'absorbé dans sa marche méditative. Le Breton évoque également le silence chez le sujet marcheur qui résulte notamment de sa présence attentive. Ce n'est donc pas au sens de l'absence de sons, mais au contraire, d'un état psychique et intellectuel. Un état qui donc mène à l'effacement de «ce qu'il y a autour de soi pour qu'il ne reste que soi » (I'Allier, 2004 p.28) Le Breton pense autrement le sujet marcheur, pour lui, c'est un être qui aspire par la marche de vivre sans toit ni loi, libre et sauvage, muni d'un flot de connaissance dans lequel il puise à chaque fois pour comprendre le monde. La marche d'Adam va dans ce sens en ce qu'il se trouve à chaque instant dans sa marche 
envahi par un «flot de mots » et de «maux ». L'on peut déduire des conclusions de Le Breton que le rapport à l'espace ne peut se faire autrement que par l'entremise du corps et des sensations.

II nous est indispensable de mettre en lumière l'errance en ce qu'elle permet également de penser la marche. Errer veut dire de se tromper, d'aller s'égarer, dans le hasard des pas. Elle peut être saisie comme un acte par lequel l'on s'écarte du droit chemin. C'est dans le sens de se perdre çà et là. De là, à dire que le marcheur errant ne suit pas d'itinéraire. Il est à préciser que le corps et l'esprit peuvent être simultanément en errance. De ce fait, l'on peut dire que l'errant est celui qui s'abandonne à un mouvement que l'on pourrait qualifié de spontané, de lâcher prise. Dans l'errance, le déambulateur « ressent, conçoit, fouille, imagine, pense la substance des choses » (Carpentier, 2004, p. 48). II nous est manifeste que le sujet pensant trouve sa définition dans ces verbeslà, l'errant est un individu qui pense soi et le monde.

Ajoutons que le sujet déambulateur est désœuvré en ce qu'il y a chez lui, ce qu'André Carpentier nomme « une névrose de passage » (Carpentier, 2004, p. 49), cela fait qu'il ne peut qu'être dans cet état de désœuvrement. De surcroit, il est constamment soumis à une «pulsions scopique et gnostique » (De Certeau, 1980, p. 155) une pulsion qui fait qu'il observe et tend à percer les mystères. Le souci du dérivant, outre celui de marcher, est d'observer, de libérer son regard et donc ses sens pour en fin libérer son esprit. Et le philosophe de définir la déambulation comme un procès infini d'être absent et en quête d'un propre (De Certeau, 1980, p. 188).

Selon André Carpentier, l'écrivain déambulateur tente de mettre en lumière ses lieux de passage en puisant dans « ses observations, dans sa sensibilité, dans ses travers, dans sa mémoire, dans son savoir, mais aussi dans la matérialité même du lieu » (Carpentier, 2004, p. 53) C'est un être qui est bien doué dans la fouille par l'écriture. En d'autres termes, il est constamment en quête de ce petit quelque chose qui déclenche l'écriture.

\section{Marche et métamorphose de soi}

De nombreuses études ont mis en lumière la marche entre corps et esprit. Elle est notamment comprise comme étant « un appel à la métamorphose de soi » (Le Breton, 2000 , p. 164). On peut citer également l'apport de Rebecca Solnit qui voit qu'idéalement, 
marcher est un état où l'esprit, le corps et le monde se répondent. Ils sont indissociables du fait que l'acte de marcher est un acte profondément attelé à l'acte de penser en cela que dans la marche, esprit et corps sont dans un dynamisme explicite. C'est dans cet esprit que David le Breton avance l'idée que la marche donne naissance au sens et à la sensation. Et l'anthropologue de poursuivre que la marche fait plonger le marcheur dans « une forme active de méditation sollicitant une pleine sensorialité » (Le Breton, 2000, p. 11). Selon lui, c'est par la marche que l'homme acquiert la faculté proprement humaine de donner du sens au monde. II présente cette pratique comme acte par lequel I'homme crée, construit le monde.

Si l'on veut comprendre la pratique de la marche, il est indispensable d'évoquer le rapport entre corps et esprit. C'est notamment dans la marche que s'anime l'esprit et le corps. Ainsi comme le stipule André Carpentier : « passer c'est penser, car le corps et la tête agissent en complices dans le rapport au lieu » (Carpentier, 2004, p. 48). En cela que l'homme qui s'adonne à la marche laisse l'espace extérieur percer son esprit ce qui signifie qu'un pacte peut exister entre l'esprit du marcheur déambulateur et le monde perçu. Ce postulat est mis en évidence par le phénoménologue Merleau-Ponty : «le monde perçu [...] est l'ensemble des chemins de mon corps » (Merleau-Ponty, 1964 p. 295) ce rapport est donc saisi par le phénoménologue comme entrelacs de relations du corps à l'espace qui est constamment sous le pouvoir de l'esprit.

On peut dire que partir en marche est pour Adam d'abord une manière de se connaitre soi-même. L'épreuve de la route lui offre l'occasion d'une expérience qu'il juge nécessaire pour se cerner comme individu. Sa marche apparait à la fois comme quête et fuite. En effet, il est pris à la fois dans un mouvement simultané d'éloignement et d'approche d'un lieu. Il fuit la ville et la vitesse qui fonde son quotidien, pour s'engager d'arrache-pied dans une longue marche en direction d'Azemmour, lieu des ancêtres. Si Adam fuit la vitesse ce n'est pas seulement parce qu'elle lui cause des « maux », c'est aussi du fait qu'il aspire par cet acte « s'écarter du droit chemin, le chemin du prévisible, du donné d'avance comme sûr » (Carpentier, 2004, p. 46 ) on peut dire qu'Adam s'écarte du droit chemin par le fait qu'il abandonne tout, sa femme, son travail et son chat. « J'ai perdu mon travail, mon chat, ma femme... Leur sacrifice ne sera pas vain... » (Laroui, 2014 , p. 82). La marche ne stimule pas seulement son corps, elle agit aussi sur son esprit au point de se livrer à de multiples réflexions philosophiques. La vie urbaine et la vitesse qui la fonde devient pour Adam menaçante et inquiétante, elle attise le « flot de mots » qui le submerge dès qu'il se met en marche. On peut dire que notre sujet marcheur 
se vide l'esprit de logorrhée extraordinaire ou de fragments de littérature française qui lui cause des « maux » et par là même, lui complique la vie. En effet, au fil des pas, il se diverse de ces mots, on peut dire qu'il se mute du fait que ces mots portent en eux une identité, celle d'un francophone agnostique. La suite du récit montrera qu'il restera agnostique, toutefois, il réactive des ressources culturelles qu'il n'aurait jamais dû délaisser.

Le personnage dans sa marche pousse son corps en dehors des limites qui lui ont été tracées. Il sait que son chemin est semé de crevasses qui lui causerait peut être des douleurs et des souffrances mais lui, il vise la connaissance de soi dans l'intention d'atteindre une renaissance. C'est ainsi qu'il ne se focalise pas sur la douleur « les maux » pour ne pas s'anéantir. On peut affirmer que le flot de mots auquel la marche donne naissance aide à la connaissance de soi. C'est souvent un vers poétique qui accorde des repères au marcheur. Qui plus est, ces vers poétiques sont à l'origine de ses maux, en marchant, Adam les met en dehors de soi ce qui le mène à se purifier et renaitre. Cela est susceptible d'apaiser ses douleurs.

Au centre de sa marche, il y a le corps, l'identité du voyageur qui, comme l'exprime Albéric d'Hardivilliers, relate sa propre expérience et supporte « le vertige qu'il y a à imaginer la saisissante nouveauté qui s'offre à nos yeux » (d'Hardivilliers. 2009, p. 24) les pas du marcheur attisent l'esprit qui ne cesse de fouiller la mémoire. II ne peut fuir ses pensées, car il parait bien qu'elles sont profondément enracinées et attachées à ses pas. De là, à dire que la marche n'est pas seulement produit du corps, elle anime également l'esprit. On peut aussi avancer l'idée selon laquelle la marche est nécessaire pour Adam, elle lui est salvatrice, c'est elle qui peut le guérir de ses maux d'esprit. Qui plus est, Laroui rejoint le Philosophe Allemand Nietzsche qui considère que « seules les pensées que l'on a en marchant valent quelque chose » (Nietzsche. 1974, p.19) c'est ainsi qu'Adam marche et pense sa vie pour se faire à la fin de nouvelles idées, un nouveau sens à sa vie, un sens qui pourrait apaiser son vertige métaphysique.

Ce faisant, Adam ne cesse de se poser la question « qui suis-je? », interrogation importante qui conforte l'idée que le protagoniste est bien en tribulations de l'esprit. On peut dire aussi qu'il tend par la marche de répondre à cette question du simple fait que dans la marche, il y a réflexion. La marche anime le corps et l'esprit. C'est seulement là où la marche s'arrête que le flot de mots s'arrête. Il y a chez Adam un dialogue de l'âme avec elle-même dans l'intention de donner sens à sa vie. Il nous est clair qu'un 
effort de conscience est attaché à sa marche en ce que sa pensée et l'état d'âme de son être sont profondément liées à son mouvement. C'est là, un voyage dans son monde intérieur sur soi-même dans l'intention de percer les mystères de son être. Au juste, le flot de mots qui submerge Adam dans la marche peut être saisi comme prise de conscience sur son être. La marche comme quête de soi peut donc être saisie au travers de la prise de conscience de sa liberté par rapport au mode de vie de la modernité. Le fait d'abhorrer la vitesse donne à penser qu'Adam aspire par-là même de s'identifier autrement. C'est donc une quête qu'il mène jusqu'au bout. La pratique de la marche et de la quête de soi n'est jamais achevée, Adam restera toujours en quête d'une vérité du fond intérieur. Si Adam décide de ne plus aller en vitesse et aller à la vitesse des ancêtres, c'est bien pour devenir ce qu'il est, un Homo sapiens qui n'a jamais dépassé la vitesse du cheval au galop. Devenir ce que l'on est peut être compris comme révolte face à la modernité et la machination qui la fonde. Dès lors, le vertige qui le frappe dans l'avion supersonique en dessus de la mer d'Andaman le bouleverse, le rend à sa nature, celle des ancêtres.

\section{La marche entre corps et esprit}

Dans les tribulations du dernier Sijilmassi, Adam en marcheur parcourt la ville souvent dans ses absences, perdu dans le labyrinthe que forment ses pensées. En cela que l'esprit est en plein activité. Adam affirme dans ce sens : « ça m'arrive, oui, je me perds dans mes pensées... comme dans un labyrinthe » (Laroui, 2014, p. 66). Qui plus est, le labyrinthe apparait comme «l'une des métaphores les plus aptes à représenter la complexité du monde contemporain et la confusion qui en découle. Tout est devenu labyrinthique » (Gervais, 2002, p. 13). II nous est manifeste qu'Adam est saisi par une sensation d'incertitude et d'ambiguité. Le récit montre que son esprit est constamment en état d'absence, absent car pensant jusqu'à entrer en «transe ». Ce qui fait de lui un être qui « oscill(e) entre le vide, le néant » (Laroui, 2014. p. 50). Cela est dû au fait qu'il ne sait plus qui il est, il peine à faire sortir ce qui ronge son esprit à savoir les mots qui lui sont « maux ». II s'exalte notamment de répondre à l'appel de la marche après la consultation du psychiatre sur la nature de ses absences et le changement radical auquel il a opté.

Comme en transe, exalté, il sortit, dévala les escaliers, ouvrit la porte de l'immeuble et se mit en marche. Demain, dès l'aube, à l'heure où blanchit la campagne, / Je partirai. C'était infernal. II se prit la tête à deux mains, 
serra violemment. Peine perdue : I'ordalie des mots continuait. Des mots, des maux. (Laaroui, 2014, p. 78)

Une fois en marche, l'esprit se mit en marche, ce qui montre que le corps et l'esprit s'activent simultanément. Dans cette optique, un vers baudelairien résonne dans son esprit actif, un vers traduisant l'appel de l'ailleurs : « Demain, dès l'aube, à I'heure où blanchit la campagne, / Je partirai » (Laroui, 2014, p. 78). Adam se met en marche vers un ailleurs. Là où il n'y a pas de vitesse, là où la vie est à la mesure de son ancêtre HomoSapiens.

Ajoutons que ce flot de mots est pour Adam un flot de « maux » la raison pour laquelle : il se prit la tête à deux mains, serra violemment. Ce dynamisme de l'esprit qui s'active au fil des pas traduit explicitement les incertitudes et une crise identitaire qui atteignent le marcheur. Ces « mots » ou ces « maux » ne résultent que des incertitudes et du dédoublement qui survient chez Adam. Les tribulations de l'esprit soulignent la nécessité d'une métamorphose chez le protagoniste. Dans ce sens, on peut citer l'épisode où il ne sait plus qui il est « un Robespierre ou un Danton ». Le parcours erratique l'a introduit dans une incertitude et une ambiguïté qui sont apparemment produit du dédoublement du sujet marcheur. Dans le même ordre d'idée, le sujet marcheur apparait comme saisi par un sentiment de perte d'identité. C'est ce que met en lumière David le Breton qui voit que la marche se veut une pratique où il y a perte de repères et quête de soi. Plusieurs passages dans le roman en témoignent manifestement à titre d'évidence, ce que le narrateur évoque comme étrange :

C'était étrange. Il y avait maintenant deux hommes en lui (deux démons ?). L'un, ardent, électrisé, fiévreux (et c'était lui qui menait son corps ( "Tu trembles, carcasse... »)) ; l'autre calme et froid, mais impuissant à contrôler son corps galvanisé. Deux hommes. Danton et Robespierre. Deux faces d'une même pièce... Ah ! ça ira, ça ira, ça ira... «Tu tremblerais bien davantage si tu savais où je vais te mener!» (Laaroui, 2014, p. 79)

Notre sujet marcheur est donc en proie à un dédoublement, deux démons logent en lui, l'un est ardent, il contrôle son corps, l'autre est explicitement impuissant face au corps galvanisé. C'est ainsi qu'un Danton et Robespierre logent en lui. Ce passage mérite d'être commenté autrement à savoir que le démon impuissant est bien le francophone en ce qu'il se vide de tous les mots, vers et textes littéraires, philosophiques, 
bref, il est forcé par le démon ardent de se débarrasser de l'homme moderne qu'il était en faveur du marocain authentique, celui qui aime les textes des philosophes andalous à l'instar de : «Hayy Ibn Yaqzân, d'Ibn Tofayl » «Traité décisif d'Ibn Rochd ». Sa découverte est frappante du fait que l'agnostique qu'il était n'a pas été secoué et que le rationalisme européen doit beaucoup à ces philosophes.

De là, à dire que notre sujet marcheur est en plein trouble du fait que ce dédoublement hante la psyché du sujet marcheur. Ainsi, le récit montrera que plus il persiste dans sa marche, plus son trouble augmente et ne prend fin qu'à l'arrivée à la terre natale Azemmour. Il est à noter que le rythme de l'écriture est d'une extrême lenteur du fait que chargé de digressions. En effet, le texte est jonché de références philosophiques et poétiques. Cela donne comme corolaire l'idée que l'acte de marcher a de multiple similitudes avec l'acte d'écrire.

Ajoutons que la marche suggère l'effort d'Adam de diminuer et les maux de l'esprit et le flot de mots qui submerge son esprit. C'est dans la marche qu'il aspire diminuer le doute ontologique ou le vertige métaphysique qui règne sur son être. En fait, à maintes reprises, le protagoniste exprime explicitement ce sentiment de perte d'identité, de déracinement. C'est notamment le cas dans : «Qui suis-je ? » (Laroui, 2014, p. 89/ 154). «Suis-je devenu un gros cafard ?» (Laroui, 2014, p. 103). « Avion, altitude, vitesse... "Qui suis-je ? », « Que fais-je ici ? », etc » (Laroui, 2014, p. 154). « Qu'est-ce qui m’arrive ?» (Laroui, 2014, p. 193). «Toujours en français. Pas une seule phrase de Mutanabbi ou de Chawki, pas un seul verset du Coran. Qui suis-je ? » (Laroui, 2014, p. 78). Ce questionnement ou cette crise des mots hâte sa marche vers le sud, lieu qu'il qualifie d'espace d'apaisement. En fait, ce à quoi Adam aspire, c'est bien à quitter la ville, lieu de vitesse, vu comme profane, lieu des « extraordinaires logorrhées » (Laroui, 2014, p.78) vers un lieu sacréà ses yeux, celui des ancêtres où il pourrait apaiser l'entrechoquement des phonèmes qui se produit dans son esprit. Il est bien conscient que c'est uniquement par la marche qu'il peut s'arracher à cette crise identitaire, à ce « flots de mots » et à ces questions ontologiques.

Plus loin dans le récit de Laroui, l'esprit du protagoniste connait l'apaisement une fois en dehors de Casablanca: « Le cercle d'acier qui opprimait sa poitrine à Casablanca, au réveil, avait disparu » (Laroui, 2014, p. 86) c'est donc en dehors de cette immense cité que les sensations ou les maux se disparaissent. En d'autres termes, dans le cas d'Adam, la marche apparait comme une guérison. C'est elle qui lui procure la distance 
d'abord physique et puis morale indispensable pour guérir la crise des «mots » et des " maux ». Sa crise identitaire et le questionnement auquel elle donne naissance se voient apaisés une fois en dehors de Casablanca. De là, à dire que la marche lui est salvatrice de l'épiphanie vécue quelques part en dessus de la mer d'Andaman.

D'après cela, on peut affirmer que le corps d'Adam est en errance aussi bien que sa pensée. C'est là une caractéristique du marcheur déambulateur. Cela a comme corolaire le fait que l'on passe chez Adam de la marche, à la parole et à la pensée. Ce qui signifie qu'il erre non seulement dans le monde extérieur mais également dans son monde intérieur.

la marche est ainsi construite, accompagnée d'une pensée en plein dynamisme, un esprit aussi éveillé qui fait que le sujet marcheur se perd dans sa culture aussi riches et ses connaissances aussi vastes. Cela va de soi avec la marche telle que représentée dans le récit de Laroui à savoir qu'Adam marche avec un esprit profondément actif de même qu'une culture et des connaissances lui viennent en flot. En cela que les textes et fragments poétiques autrefois lus montrent qu'il marche avec un bagage de culture. Cela dit, ce sujet déambulateur se promène dans sa propre mémoire. Tous ces mots qui « s'entrechoquent » dans son esprit éveillé ne résultent que de la mémoire qui s'active au fil des pas.

\section{Marche et révolte}

Stricto sensu, la pratique de la marche s'inscrit également dans l'espace urbain. Le marcheur s'y met en lutte dans l'intention de transgresser les normes établies. Ainsi, son ambition est celle d'aller au-delà des « espaces civilisés » et ce, pour une appréhension plus ample du monde. En d'autres termes, La marche signifie révolte contre les normes établies par la société du fait que cet acte constitue une piste favorisée pour retisser les liens perdus. À cet égard, ce type de marcheur, stipule François l'Allier, « se place également en position de révolte contre la culture, l'ordre urbain établi (par exemple la circulation des voitures, les feux de signalisation, etc.) » (L'Allier, 2004, p. 26). II nous est clair que la marche constitue une critique en mouvement de la modernité. Si Sylvain Tesson entend fuir le monde numérisé et s'oppose au règne de la prévisibilité, Adam, pour sa part, Après l'épiphanie vécue en dessus de la mer d'Andaman, il se révolte contre la vitesse qui fonde le monde contemporain. Il fuit tout engin, la vitesse lui est vertige. C'est ainsi qu'il se révolte par la marche à la manière de ses ancêtres Homo 
Sapiens. Sorti de l'aéroport, il refuse de prendre un taxi. Il émet un mensonge pour se dégager de la horde des taxis jonchée à la sortie: « II se dégagea du mieux qu'il put, répétant qu'il possédait une voiture et qu'elle l'attendait au parking. Pourquoi ce mensonge ? » (Laroui, 2014, p. 9). Cela semble plus vraisemblable que sa décision de « marcher jusqu'à Casablanca » (Laroui, 2014, p. 9). Sa décision de rejoindre la ville par la marche parait être hors norme ce qui lui a valu de rentrer chez lui d'autorité encadré par deux gendarmes. C'est ainsi que sa marche commence, une marche qui par la suite fait éveiller sa réflexion sur le monde.

Dans la même optique, l'anthropologue David le Breton explique que la marche dans le monde contemporain, pourrait évoquer une forme de nostalgie ou résistance (Le Breton, 2000, p.14). Cela va de soi avec la situation du protagoniste. Sa marche peut être saisie d'abord comme nostalgie en ce qu'il se dit vouloir marcher à la manière de son ancêtre le Hadj Maati qui n'a jamais dépassé la vitesse de quatre kilomètre à l'heure. Il s'agit en somme d'une forme de résistance face au mode de vie de la modernité urbaine.

Il s'engagea sur le bord de la route, traînant sa valise à roulettes, et atteignit bientôt sa vitesse de croisière : quatre kilomètres à l'heure. Pendant sept millions d'années, aucun homo, ni erectus ni sapiens, n'avait longtemps dépassé cette allure : au regard des millénaires, il était dans la norme. (Laaroui, 2014, p. 9)

Le début du récit montre comment Adam sijilmassi a vécu une étrange épiphanie alors qu'il survolait la mer d'andaman, il se posa des questions perturbantes, on peut citer dans ce sens : « que fais-je ici ? » (Laroui, 2014, p. 154) propulsé dans l'air à des vitesses pharamineuses tandis que ses ancêtres n'avait jamais dépassé la vitesse d'un cheval au galop. Ce fut une épiphanie ayant déclenché les cogitations du protagoniste. Il comprit notamment qu'il vit la vie d'un autre et qu'il y avait quelque chose d'indigne dans cette translation de l'humain «le long d'une géodésique du monde » (Laroui, 2014, p. 6). Cela le pousse à changer de vie et à faire de la marche ce par quoi il résiste face à ce que Alexis l'Allier nomme la « déshumanisation du monde » à laquelle l'homme moderne a donné naissance. Autrement, si Adam décide de ne plus filer à l'allure du Boeing, c'est pour se tenir hors de la vie moderne. Son corps n'est plus identique au corps moderne qui à l'image de la machine, se déplace à haute vitesse. De là, à dire que le protagoniste veut par l'entremise de la marche renouer avec le corps biologique 
que le corps moderne tente d'enterrer. C'est là encore vouloir résister par le mouvement du corps qui n'est plus une activité désuète. C'est ainsi qu'il pense autrement son rapport à la machination qui a fait que la marche représente une action anachronique et marginale dans un monde où l'on se déplace en automobile. Plus loin dans le récit de Laroui, Adam se remit en marche vers la ville d'Azemmour :

Il ne songea pas un instant à lever le bras et à arrêter un des grands taxis blancs qu'il voyait filer sur la route et qui lui adressaient des appels de phares. Non, c'était à pied, à l'allure de l'homme, qu'il lui fallait retourner vers le long boyau noir. J'ai l'apparence d'homme pour prouver que le monde est fait à ma mesure. (Laaroui, 2014, p. 82)

Ajoutons également que la marche dans la ville se veut une nouvelle manière de « changer d'existence » (Le Breton, 2000, p. 11) de sortir de soi et de se mettre loin de tout engin et donc de la vie moderne pour ainsi mieux rentrer en soi ou aller chez soi.

Plusieurs faits attestent que la marche initiée à la sortie de l'aéroport de Casablanca apparait hors norme. En effet, les gens rencontrés en route sont sidérés par le choix d'Adam. Cela donne à penser que le fait de ralentir dans le contexte de la vie moderne choque et apparait comme une transgression. Marcher est donc une activité désuète pouvant frapper de stupéfaction malgré le fait que I'humanité n'a fait que marcher jusqu'à un temps très récent.

Cela se produit à titre d'exemple à la sortie de l'aéroport. La route est si longue et la marche d'Adam apparait comme étrange conduite. Tel est son objectif, marcher jusqu'à Casablanca. Tout au long de la route, les automobilistes s'arrêtent lui proposant de le déposer, à chaque fois le refus est rendez-vous: «II répondit d'un ton ferme : — Non, merci. » (Laroui, 2014. p. 10) « — Je vais à Casablanca. Mais j'ai envie de marcher. Merci pour l'offre. » (Laroui, 2014, p. 10) — Je vais à Casablanca. À pied. Allez-y, votre femme s'impatiente » (Laroui, 2014, p. 15) ces réponses sont difficilement acceptables parce qu'étant hors norme. « Personne, tu entends bien, personne ne va à pied à Casa à partir d'ici (...) Cette foutue ville est déjà assez grande pour t'épuiser, toi et ta valise ». «On ne va pas à pied à Casa. C'est impossible. Ça n'existe pas » (Laroui, 2014, p. 11). De là, à reconnaitre que la marche d'Adam est transgressive, choquante et hors norme. 


\section{Marcher d'un lieu profane à un lieu sacré}

Il nous parait important d'éclairer la dimension spirituelle de la marche en ce qu'elle traduit le passage du marcheur d'un lieu profane à un lieu sacré. De là, à dire que le monde se scinde en deux. Ainsi, le marcheur pèlerin passe d'un lieu profane qui est manifestement la ville, lieu où le malaise des maux et des mots atteint le sujet marcheur, le trouble, au point de songer dans une marche pénible et d'extrême lenteur. Notre sujet marcheur entend à la manière d'un pèlerin se purifier et renaitre en marchant avec des cailloux dans les souliers. Il semble évident que le marcheur s'efforce d'augmenter la pénibilité du voyage en direction de son lieu natale perçu comme sacré et comme espace d'apaisement. Pour notre sujet marcheur, il est indispensable de marcher jusqu'à l'arrivé sinon c'est la mort qui l'attend « Marche ou crève » (Laroui, 2014, p. 88). II nous est manifeste que l'itinéraire que suit Adam est bien similaire à celui d'un pèlerin en ce qu'il décide de donner un tout nouveau sens à son existence d'homme, il laisse tout derrière lui et revient vivre dans sa terre natale. Autrement, il abandonne la ville, lieu des mots et des maux de l'esprit pour aller en quête de ses racines et de ses origines. Il est ainsi en quête d'un ailleurs où il peut renaitre, un ailleurs qui lui est sacré, à savoir celui des ancêtres, un ailleurs où il n'aurait point de trouble. C'est ainsi qu'il se met à rude épreuve, celle d'un chemin semé d'embuches, ceci fait penser aux pèlerins qui s'efforcent d'augmenter la pénibilité du voyage. Pour atteindre ce qu'il nomme le long boyau noire ou encore ce lieu de délivrance, II se livre à une marche lente et pénible: " c'était à pied, à l'allure de l'homme, qu'il lui fallait retourner vers le long boyau noir. J'ai l'apparence d'homme pour prouver que le monde est fait à ma mesure» (Laroui, 2014, p. 82). À cet égard, si, dans la marche, le corps peut être en plein dynamisme, l'esprit est aussi soumis à rude épreuve. Il est toujours sous le poids des « mots » et des « maux ». Ce voyage pénible et lent conduit donc à l'épuisement du corps et de l'esprit.

Il était épuisé. " Couchons-nous sur la terre, et dormons. » La terre, il lui sembla qu'elle était boueuse (cette boue est-elle faite de mes larmes? Je perds la tête...). II fit quelques pas et s'assit sur le seuil d'un café clos pour la nuit, posa la tête sur ses bras croisés sur ses genoux et sombra dans un sommeil agité. (Laaroui, 2014, p. 85)

Selon l'anthropologue David le Breton, le sujet marcheur « n'élit pas de domicile dans l'espace mais dans le temps » (Le Breton, 2000, p. 26). De ce fait, ce n'est pas les frontières dans l'espace qui l'arrêtent mais bien le cycle du jour et de la nuit qui scinde 
son cheminement. C'est ainsi qu'Adam, une fois épuisé la nuit, il songe dans un sommeil au seuil d'un café. C'est donc les contraintes temporelles qu'affronte le marcheur dans son chemin. On peut dire également que la marche met en péril le sujet marcheur, c'est notamment le fait quand le temps l'arrête pour une nuit aux environs de Bir Jdid:

Je te conseille de ne pas rester ici. Des chiens sauvages rôdent dans les parages. S'ils s'enhardissent, ils pourraient bien s'attaquer à toi. Tu ne veux quand même pas finir déchiqueté par des chiens? Et si les gendarmes te découvrent ici, ils t'emmèneront... (Laaroui, 2014, p. 85)

C'est ainsi que marcher devient synonyme d'invention. Dans ce corps à corps avec le monde, notre sujet marcheur entend « se tenir hors». C'est ainsi que marcher devient également synonyme d'exister, ce qui veut dire résistance face à la vie moderne et la vitesse qui la fonde si non la vie s'enlise.

\section{Conclusion}

En guise de conclusion, il ressort de l'analyse de ce roman philosophique que la marche permet au protagoniste de se métamorphoser. Tout au long de ses tribulations, il se vide l'esprit de tous les textes et fragments poétiques de littérature française qu'il avait auparavant mémorisé. Ce qui conforte l'idée que dans la marche, le corps et l'esprit sont en plein dynamisme. C'est ainsi que se métamorphose notre sujet marcheur en redevenant le marocain authentique qu'il n'aurait jamais dû délaisser. Cela ne s'est pas fait sans malaise, sans vertige du fait que le flot de mots ou ce qu'il qualifie de logorrhée s'accompagne de «maux ». Ce qui conforte l'idée que la marche ouvre la voie aux sens et à la sensation. On comprend également que marcher c'est penser en ce que dans sa marche, le protagoniste se dit se perdre dans ses pensées comme dans un labyrinthe. De là, à reconnaitre que dans la marche d'Adam, il y a perte de repères et quête de soi exactement et comme le stipule Le Breton. La sensation de dédoublement et le malaise identitaire qu'il y a chez lui en témoignent manifestement. La marche inscrite dans la métropole apparait dans le roman comme une lutte et transgression des normes de la vie moderne, la décision de marcher devient une folie parce que le fait de ralentir dans la vie moderne choque, constitue une transgression des normes établies par la modernité. La marche apparait également comme résistance en ce que le sujet marcheur résiste face aux appels des taxis et automobilistes. Personne ne comprend le pourquoi de sa décision à tel point que la gendarmerie le ramène d'autorité chez lui. La marche 
d'Adam traduit également un désir qui est celui de passer d'un lieu qu'il juge profane à savoir la ville, espace " des mots/ maux » et de son malaise identitaire, vers un lieu qu'il juge sacré, le sud en dehors de la ville, qualifié d'espace de son apaisement. En cela que l'espace dans le roman se scinde en deux lieux. On comprend également à travers ce roman fait de tribulations que le sujet marcheur n'a pas de domicile dans l'espace en ce qu'il ne s'arrête guère par les frontières spatiales, c'est plutôt le cycle du jour et de la nuit qui scinde son cheminement. On peut déduire également de ce roman fait de tribulations que ce marcheur contemporain entend se « tenir hors » par la marche, ce qui fait de cet acte un synonyme d'exister, que l'on peut saisir comme résistance à la vie moderne si non la vie du héros s'enlise.

Évaluation : Évaluation anonyme par des pairs extérieurs.

Conflit d'intérêts : L'auteur n'a aucun conflit d'intérêts à déclarer.

Subvention : L'auteur n'a reçu aucun soutien financier pour ce travail.

Peer-review: Externally peer-reviewed.

Conflict of Interest: The author has no conflict of interest to declare.

Grant Support: The author declared that this study has received no financial support.

\section{Bibliographie}

Breton, L. D. (2000). Éloge de la marche. Paris : Métailié.

Carpentier, A. (2004) « Huit remarques sur l'écrivain en déambulateur urbain ». Dans Les écrivains déambulateurs :

Poètes et déambulateurs de l'espace urbain. Article d'un cahier Figura. En ligne sur le site de l'Observatoire de l'imaginaire contemporain. <http://oic.uqam.ca/fr/articles/huit-remarques-sur-lecrivain-en-deambulateururbain>. Consulté le 2 Janvier 2021.

Certeau, M. D. (1994). L'invention du quotidien (Arts de faire 1), Paris, Union générale d'édition, coll. « 10-18 » Certeau, M. D. (1987). Histoire et psychanalyse entre science et fiction. Paris: Gallimard.

Gervais, B. (2002). « Le labyrinthe et l'oubli. Fondements d'un imaginaire ». Dans L'imaginaire du labyrinthe. Article d'un cahier Figura. En ligne sur le site de l'Observatoire de l'imaginaire contemporain. <http://oic. uqam.ca/fr/articles/le-labyrinthe-et-loubli-fondements-dun-imaginaire>. Consulté le 2 Janvier 2021.

Hardivilliers, d .A. (2009). L'écriture de l'ailleurs. Petits propos sur la littérature nomade. Paris : Transboréal.

L'Allier, A. (2004) «La déambulation, entre nature et culture ». Dans Les écrivains déambulateurs : Poètes et déambulateurs de l'espace urbain. Article d'un cahier Figura. En ligne sur le site de l'Observatoire de l'imaginaire contemporain. <http://oic.uqam.ca/fr/articles/la-deambulation-entre-nature-et-culture>. Consulté le 7 janvier avril 2021. 
Laroui, F. (2014). Les tribulations du dernier Sijilmassi. Paris : Julliard.

Merleau-Ponty, M. (1964). Le visible et l'invisible. Paris : Gallimard.

Nietzsche, F. (1974). Le crépuscule des idoles. Paris : Gallimard.

Solnit, R. (2002). L'art de marcher. (trad. de l'américain par Oristelle Bonis), Paris : Actes Sud. 